\begin{tabular}{|c|c|}
\hline Citation & $\begin{array}{l}\text { Maarten Tytgat, Niels Van Thienen, Patrick Reynaert, (2015), } \\
\text { Title } \\
\text { Analog Integrated Circuits and Signal Processing, vol } 83 \text { (issue 1), pp 55-64. }\end{array}$ \\
\hline Archived version & $\begin{array}{l}\text { Author manuscript: the content is identical to the content of the published } \\
\text { paper, but without the final typesetting by the publisher }\end{array}$ \\
\hline Published version & $\begin{array}{l}\text { insert link to the published version of your paper } \\
\underline{\text { http://link.springer.com/article/10.1007/s10470-015-0510-6 }}\end{array}$ \\
\hline Journal homepage & $\begin{array}{l}\text { insert link to the journal homepage of your paper } \\
\text { http://link.springer.com/journal/10470 }\end{array}$ \\
\hline Author contact & $\begin{array}{l}\text { your email patrick.reynaert@esat.kuleuven.be } \\
\text { your phone number + } 32(0) 16321878\end{array}$ \\
\hline
\end{tabular}

(article begins on next page) 


\title{
A 90-GHz Receiver in 40-nm CMOS for Plastic Waveguide Links
}

\author{
Maarten Tytgat, Niels Van Thienen, and Patrick Reynaert
}

Received: date / Accepted: date

\begin{abstract}
Plastic waveguide links are proposed as a costfriendly, light-weight and EMI-robust alternative for copper and optical interconnects for Gbps data transfer up to $10 \mathrm{~m}$. Measured properties of the rectangular polypropylene waveguide such as dielectric loss and bending loss are reported. The design and measurements of a $90-\mathrm{GHz}$ injectionlocked ASK receiver in 40-nm CMOS are presented. The link is demonstrated with the receiver chip and a plastic waveguide with lengths up to $9 \mathrm{~m}$. The highest achieved bit rate is $9 \mathrm{Gbps}$ for a distance of $60 \mathrm{~cm}$ and $2.5 \mathrm{Gbps}$ for a distance of $9 \mathrm{~m}$ with a BER $<10^{-12}$. With a power consumption of $50 \mathrm{~mW}$ from a $0.9 \mathrm{~V}$ supply, the best FOM is $2.2 \mathrm{pJ} / \mathrm{bit} / \mathrm{m}$.
\end{abstract}

Keywords CMOS - Dielectric waveguides · Millimeter wave $\cdot$ Plastic $\cdot$ Receivers

\section{Introduction}

The pursuit of high bit rates in data communication systems has driven researchers to use even higher carrier frequencies reaching the millimeter wave and even the terahertz range. The price to be paid for the large bandwidth is the increasing power loss of the RF signal. Fig. 1 compares the loss in

\section{Tytgat}

Alcatel-Lucent, Bell Labs

Copernicuslaan 50, 2018 Antwerpen, Belgium

N. Van Thienen

KU Leuven, ESAT/MICAS

Kasteelpark Arenberg 10, 3001 Heverlee, Belgium

P. Reynaert

KU Leuven, ESAT/MICAS

Kasteelpark Arenberg 10, 3001 Heverlee, Belgium

Tel: +32163210 77

Fax: +3216321975

E-mail: patrick.reynaert@esat.kuleuven.be

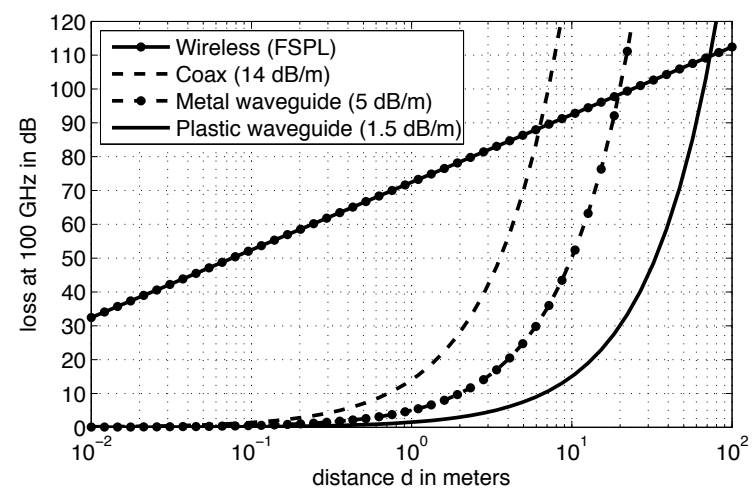

Fig. 1 Loss of different millimeter wave channels at $100 \mathrm{GHz}$.

four millimeter wave channels at a frequency of $100 \mathrm{GHz}$ : a wireless link, a metal waveguide, a coaxial cable and a dielectric waveguide.

In the case of a wireless link, only the free space path loss (FSPL) is taken into account since atmospheric attenuation can be neglected for the considered distances. The FSPL is quadratic versus distance and is already extremely high for small distances. At $1 \mathrm{~m}$, it is more than $70 \mathrm{~dB}$. This loss can only be compensated by using high RF powers and directional antennas, something which is hard to achieve in CMOS technologies. The loss in a coax cable, metal waveguide or plastic waveguide are exponential with length, and have a fixed $\mathrm{dB} / \mathrm{m}$ value. Their loss is acceptable for lengths up to several meters.

Coax cables are used for measurements up to $110 \mathrm{GHz}$ with a loss of $14 \mathrm{~dB} / \mathrm{m}$ [1]. They become increasingly expensive when the operating frequency rises. Also the connectors become very critical and expensive.

Metal waveguides are also popular in lab environments. They exhibit lower loss than coax cables, $5 \mathrm{~dB} / \mathrm{m}$ [2], thanks to their larger current-carrying area, but also become expensive 


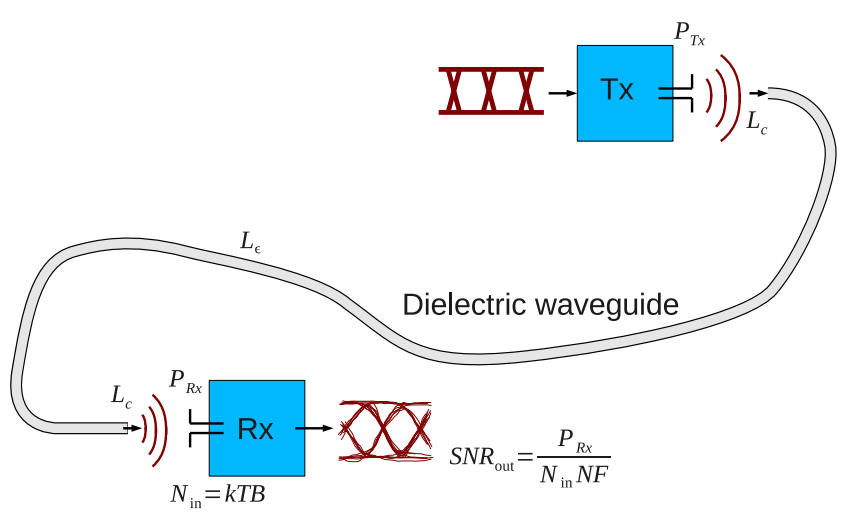

Fig. 2 Concept of a millimeter wave plastic waveguide link with transmitter and receiver ICs with on-chip antennas.

for high frequencies. Moreover, they are rigid and require special parts such as S-bends, 45 degree bends and tapers to connect different pieces of equipment, which introduces extra losses.

A possible solution to these issues can be found in dielectric waveguides, with a measured loss of only $1.5 \mathrm{~dB} / \mathrm{m}$. Glass and plastic waveguides have already been used for years in optical communications. However, optical networks are often expensive and complex because conversion is needed between the electrical and the optical domain and alignment between the fiber and the light source or light detector is very critical.

The aim of this work is to exploit the properties of cheap plastics such as polypropylene (PP), polystyrene (PS) and high density polyethylene (HDPE) to implement a low-cost and flexible communication link. This will be implemented with millimeter wave CMOS chips at both ends to transmit and receive the signals. The concept is shown in Fig. 2. Onchip antennas are used to interface between the chips and the dielectric waveguide. This way, the use of electrical-tooptical and optical-to-electrical converters is avoided which results in reduced cost and complexity.

This work focuses on plastic waveguides with rectangular cross-sections. The dimensions are close to the wavelength of the used RF signal. Lab measurements reveal a loss in the plastic waveguide of $1.5 \mathrm{~dB} / \mathrm{m}$ at a frequency of $100 \mathrm{GHz}$. A simplified link budget calculation shows the feasibility of the plastic waveguide link in Fig. 2 for a length of $10 \mathrm{~m}$. Assume a transmitted power $P_{T x}$ of $0 \mathrm{dBm}$, a coupling loss $L_{c}$ of $10 \mathrm{~dB}$ at each side and a dielectric loss $L_{\varepsilon}$ of $15 \mathrm{~dB}$ in the waveguide. The received power $P_{R x}$ is then $-35 \mathrm{dBm}$. With a bandwidth $B$ of $10 \mathrm{GHz}$ and a receiver noise figure $N F$ of $10 \mathrm{~dB}$, an $S N R_{\text {out }}$ of $29 \mathrm{~dB}$ remains, which is sufficient to receive ASK, BPSK, OOK or QPSK with a $B E R<10^{-12}$.

The plastic waveguide link is thus suitable for bit rates up to $10 \mathrm{Gbit} / \mathrm{s}$, over distances up to $10 \mathrm{~m}$. Possible applications include high-speed data links in cars, next-generation USB, high-speed connections in data centers and so on. Nowadays

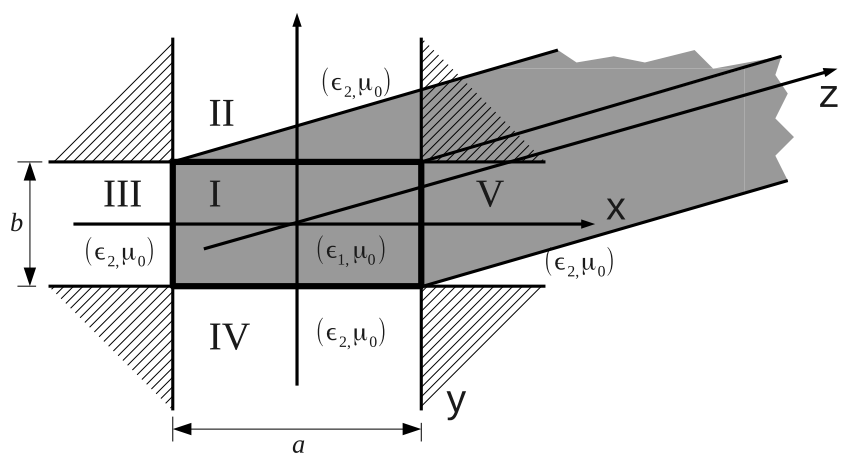

Fig. 3 Cross-section of the dielectric waveguide surrounded by a medium. For Marcatili's approximation, the fields in the shaded regions are ignored.

these applications often use copper wires. Copper connections typically have a lower loss than plastic waveguides but suffer from EMI and equalization is needed to achieve high data rates. Plastic waveguides on the other hand provide galvanic separation and their weight is much lower, a property that is especially important in automotive applications.

The receiver chip presented in this paper was published in [3]. This paper goes into more detail on the dielectric waveguide and the design of the receiver circuit.

In the next section, the properties of rectangular dielectric waveguides for millimeter wave frequencies are examined. The validity of Marcatili's approximation [4] is investigated and simulations and measurements on plastic waveguides are reported. Section 3 describes the design of the $40 \mathrm{~nm}$ CMOS receiver chip with on-chip dipole bondwire antenna. Measurements to characterize the chip performance and to demonstrate the plastic waveguide link are reported in section 4.

\section{Rectangular Plastic Waveguides}

\subsection{Wave propagation: Approximation and Simulations}

In 1969, Marcatili [4] proposed a set of approximate equations to describe the propagation of EM-waves in dielectric waveguides. This was based on the assumption that most of the power is flowing inside the waveguide, ignoring the EM-field in the shaded regions in Fig. 3. Depending on the frequency, the dimensions of the waveguide and the dielectric constant, different modes can propagate. They are divided into two groups: the $E_{p q}^{x}$ modes and the $E_{p q}^{y}$ modes. The superscript denotes in which direction the electric field is dominant. The subscript indicates the number of extrema the dominant field component has along the $x$-direction $(p)$ and the $y$-direction $(q)$. The $z$-direction is in the direction of propagation, along the waveguide, see Fig. 3. In the channel measurements, rectangular metal waveguides are used to couple signals from the source to the plastic waveguide 


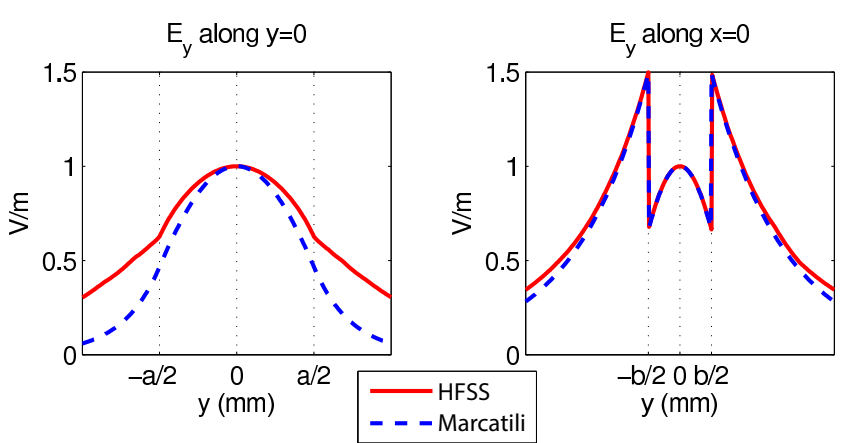

Fig. 4 Normalized $y$-component of the electric field vector at $90 \mathrm{GHz}$ in a dielectric waveguide in two cutting planes: $y=0$ and $x=0 . \varepsilon_{r}=$ 2.26, $a=2.2 \mathrm{~mm}, b=0.9 \mathrm{~mm}$. Solid lines are HFSS simulation results and dashed lines are Marcatili's approximation of the $E_{11}^{y}$ mode.

or from the plastic waveguide to the measurement equipment. Since in these metal waveguides, the used mode is $T E_{01}$, with the electric field polarized along the $y$-axis, only the $E_{p q}^{y}$ modes are excited in the plastic waveguide. Therefore, the $E_{p q}^{x}$ modes will be disregarded from here on. The fundamental mode is then the $E_{11}^{y}$ mode.

Fig. 4 shows the $y$-component of the electric field along the lines $y=0$ and $x=0$. The discontinuities at $y= \pm b / 2$ arise from the boundary condition of the normal component of the electric field vector. The $y$-component of the electric field is continuous at the boundaries at $x= \pm a / 2$. Simulation results are shown from the full-wave EM solver HFSS, as well as Marcatili's approximation of the $E_{11}^{y}$ mode. The results match quite well.

The simulated propagation constant for the same waveguide is shown in Fig. 5a. As de frequency drops, it deviates more and more from the Marcatili approximation. According to the approximation, each mode has a lower cut-off frequency but this is not seen in the simulation. This is due to the fact that the approximation is only valid as long as most of the guided power is inside the waveguide. It is seen in simulations that when the frequency drops, more and more power is actually travelling on the outside of the waveguide which means the approximation is no longer valid. Fig. 6 shows the power density on a cross-section of the waveguide. According to the simulations, at $50 \mathrm{GHz}$, only $0.8 \%$ of the total power flows inside the waveguide, which increases to $36 \%$ at $100 \mathrm{GHz}, 68 \%$ at $150 \mathrm{GHz}$ and $79 \%$ at $200 \mathrm{GHz}$. This shows that the Marcatili approximation can only be used far above the cut-off frequency it predicts based on the geometry of the waveguide, but it provides useful insight in the field distribution.

\subsection{Attenuation - Simulations and Measurements}

Fig. 5b shows the simulated attenuation constant $\alpha$ for a PP waveguide. With increasing frequency, more power is con-

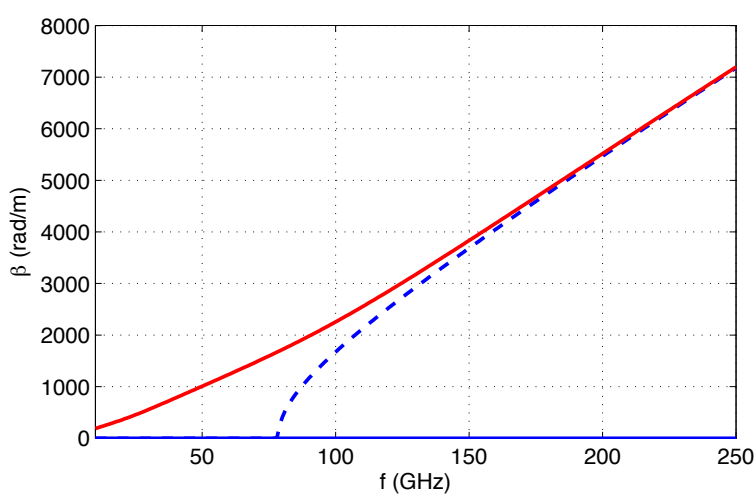

(a) Propagation constant versus frequency of the fundamental mode in a dielectric waveguide. Solid line is HFSS simulation, the dashed line is the Marcatili approximation of the $E_{11}^{y}$ mode.

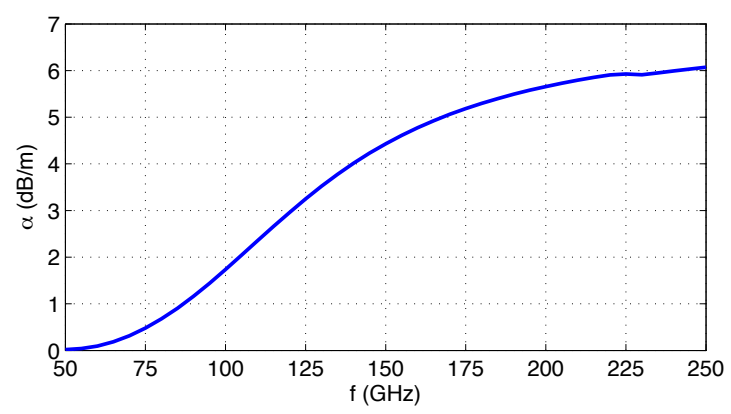

(b) Simulation in HFSS of the attenuation constant $\alpha$.

Fig. 5 Propagation constant $\gamma=\alpha+j \beta$ of a PP waveguide. HFSS simulations and Marcatili approximation of $\beta$ (a) and HFSS simulation of $\alpha$ (b). $\varepsilon_{r}=2.26, \tan \delta=0.5 \times 10^{-3}, a=2.2 \mathrm{~mm}, b=0.9 \mathrm{~mm}$.
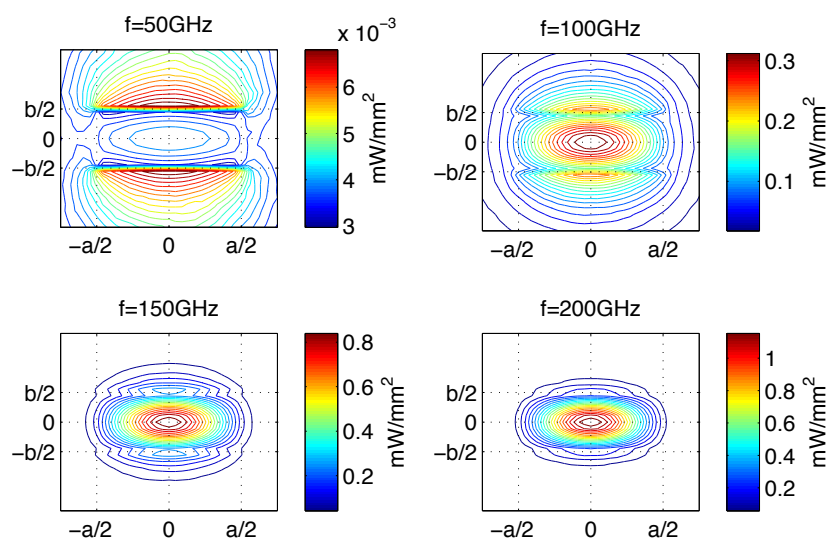

Fig. 6 Simulations in HFSS of the $z$-component of the Poynting vector. The applied power is $1 \mathrm{~mW}$. The power is more concentrated inside the waveguide for higher frequencies. $\varepsilon_{r}=2.26, a=2.2 \mathrm{~mm}, b=0.9 \mathrm{~mm}$. 
centrated inside the waveguide, which results in higher loss, since the loss tangent of the plastic is higher than that of air. At $50 \mathrm{GHz}$, the loss approaches $0 \mathrm{~dB}$, because most of the power is in the region around the waveguide, as was shown in Fig. 6. Attenuation can be measured in the lab with a network analyzer. To minimize reflections between the metal and the plastic waveguide, the tip of the waveguide is cut in a V-shape [5] and inserted in a horn antenna which is connected to the waveguide port of the frequency extender of the network analyzer. Fig. 7a shows the $S_{21}$ parameter of a $1.6 \mathrm{~m}$, a $3.4 \mathrm{~m}$ and a $9 \mathrm{~m}$ long PP waveguide. From these measurements, the loss per meter can be estimated. At $100 \mathrm{GHz}$, this is $1.5 \mathrm{~dB} / \mathrm{m}$, which corresponds well to the loss predicted by the simulated $\alpha$ of Fig. $5 \mathrm{~b}$. The measured $S_{11}$ of the waveguide is below $-10 \mathrm{~dB}$ in the entire measurement range, and therefore not represented the figures, this shows that reflections are indeed minimized. When the waveguide is bent, additional loss is introduced. This was measured by placing the waveguide in a half circular cut in a polymer foam. The foam acts nearly as air but does cause some loss. To keep this loss the same for each bend radius, the total length of the waveguide running through the foam is kept constant. The resulting measurements are depicted in Fig. 7b. As frequency drops, more power is flowing on the outside of the waveguide, so the EM-wave is less tightly bound to the waveguide and power is more easily lost in the bend as it gets re-radiated into free space. As expected, the loss increases for smaller bending radii.

\subsection{Conclusions}

Several important conclusions can be drawn from the performed simulations and measurements. Most of the electromagnetic power is flowing outside the waveguide at frequencies of $100 \mathrm{GHz}$ and below. This makes the Marcatili approximation unvalid and causes there to be no cut-off frequency. For even lower frequencies, the wave is actually no longer guided, but propagating in free space. The fact that the EM-wave is less tightly bound for lower frequencies has two important consequences. First, the dielectric loss approaches $0 \mathrm{~dB}$, since the largest part of the EM-wave is propagating in free space, with a zero loss tangent. This might seem promising, but the second consequence is that the bending losses increase severely. Both effects were confirmed by measurements.

These conclusions have a very important impact on the choice of the carrier frequency for the plastic waveguide link. A higher frequency allows more bandwidth and results in a thinner waveguide that is more flexible, with lower bending loss. On the other hand, dielectric loss increases with frequency, power generation becomes more difficult, as well as the design of the circuits.

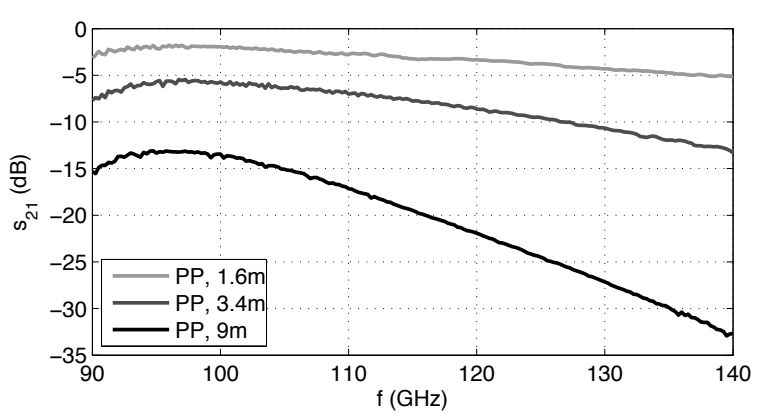

(a) Attenuation

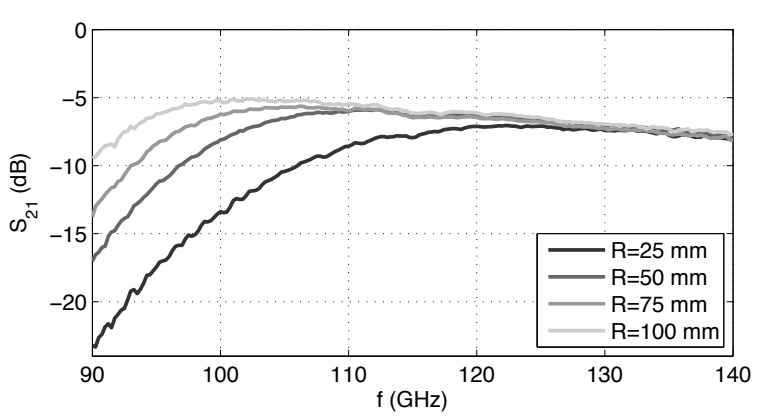

(b) Bending loss

Fig. 7 Measurements of loss in PP waveguides with fixed crosssection: $a=2.2 \mathrm{~mm}, b=0.9 \mathrm{~mm}$. The loss contains coupling loss, dielectric losses in (a) and additional bending losses in (b).

A compromise was found at $90 \mathrm{GHz}$, for which a CMOS receiver was designed, as explained in the next section.

\section{Design of the Receiver Chip}

The schematic of the complete receiver is shown in Fig. 8. It is based on the injection locking principle. When the input power of the receiver is high enough, the injection locked oscillator (ILO) is assumed to run at the same frequency as the transmitted signal and with a constant phase difference. To lock the ILO on the RF signal, the signal from the LNA is split [6]. One part is sent to the injection path, which contains an additional buffer stage. The other part goes to the bottom transistors of a double balanced Gilbert mixer. The ILO removes the modulation from the RF signal to generate the LO signal for the mixer which downconverts the signal to baseband. This is only possible when a carrier component is present in the signal like in OOK and (multilevel) ASK. Pure BPSK will not be demodulated by the receiver, since the phase of the ILO will tilt every time the phase of the RF signal tilts. The baseband amplifiers are the same as in [7]. The last stage is designed to drive the off-chip $50 \Omega$ load. 

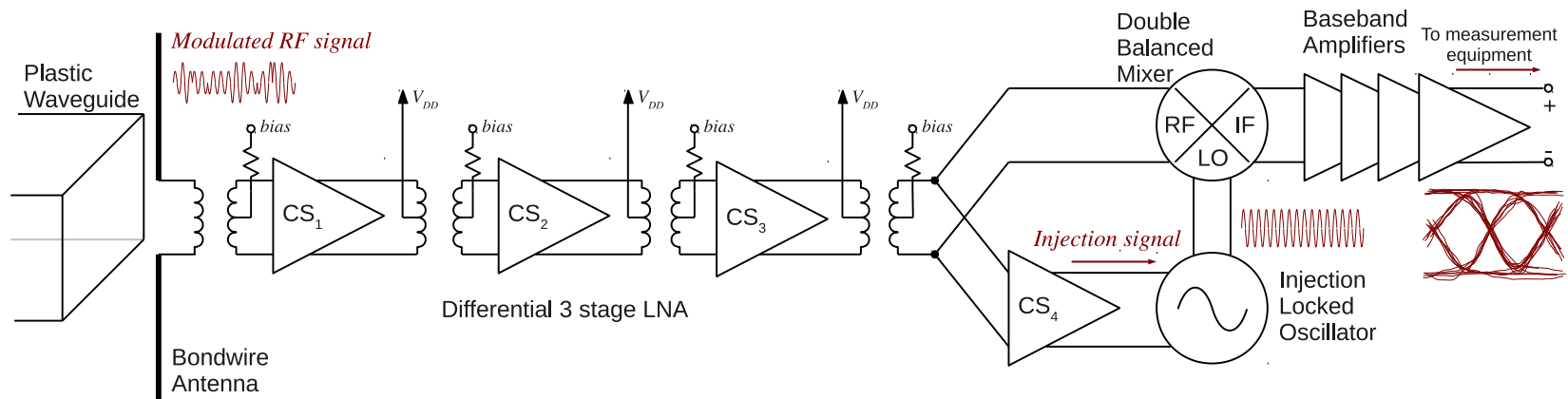

Fig. 8 Presented receiver chip, consisting of an on-chip bondwire antenna, LNA, injection-locked oscillator, double balanced mixer and baseband amplifiers.

\subsection{Low Noise Amplifier}

The LNA is a cascade of three identical transformer-coupled pseudo-differential common-source stages. The schematic is shown if Fig. 9a. Neutralization capacitance is added for reverse isolation and differential-mode stability. Increasing the reverse isolation means decreasing $S_{12}$ and thus decreasing the dependence of the input impedance on the load impedance and of the output impedance on the source impedance. This eases the matching at the input and the output of each stage. Extra resistors are added in the bias lines to improve commonmode stability. The design is similar to that of the amplifier in [8].

The common-source transistors consist of 16 fingers of $1 \mu \mathrm{m}$ and the neutralization transistors have 12 fingers of $1 \mu \mathrm{m}$. The channel lengths are minimal. The sizes are kept small to minimize DC power consumption. Since the RF power levels are rather low, large devices are not needed to ensure linear behaviour. Smaller devices are however more difficult to match [7]. The bias voltage is chosen for maximum gain and for minimum noise figure. Fortunately, both have the same optimum, for a gate voltage of $700 \mathrm{mV}$.

Transformers and transmission lines are used to match the antenna to the input of the LNA, for matching between the LNA stages and at the output of the LNA. A parallel stub is added between the output of the LNA and the power splitter to improve impedance matching. The transmission lines, the transformers, the parallel stub and the power splitter are simulated using ADS Momentum. For the common-source stages, a parasitic extraction was performed on the final layout. This, in combination with the RF-model of the transistors themselves and the Momentum results for the passive structures, gives the transducer gain curve of Fig. $9 \mathrm{~b}$ and the noise figure of Fig. 9c. The gain at $90 \mathrm{GHz}$ is $19.9 \mathrm{~dB}$ and the noise figure is $6.7 \mathrm{~dB}$.

The buffer stage in the injection path is a common-source stage like in the LNA but with smaller sizes: $W=8 \mu \mathrm{m}$ for the common-source transistors and $W=6 \mu \mathrm{m}$ for the neutralization capacitances.

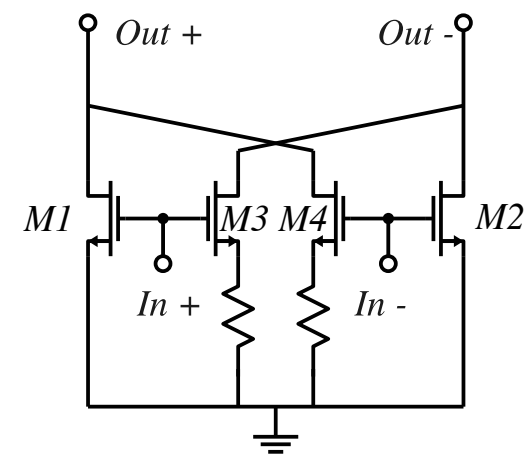

(a) Schematic of one single common-source stage, transistors M1 and M2 act as differential pair with cross-coupled transistors M3 and M4 as capacitive neutralization.

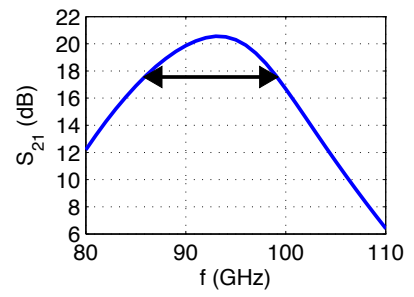

(b) Gain

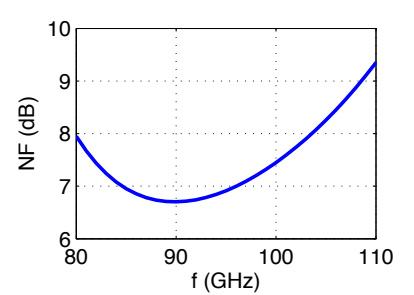

(c) Noise figure
Fig. 9 Circuit of one stage and simulated performance of the LNA.

\subsection{Downconversion Mixer}

The downconversion mixer is shown in Fig. 10a. It's a classical Gilbert cell with neutralization capacitance in the bottom pair. The pMOS transistors in the linear region act as resistive loads. The neutralization in the bottom pair of the mixer eases the impedance matching between the output of the LNA and the RF input of the mixer. A transformer could be added between the top and the bottom transistors to increase the voltage headroom on the transistors, but even with a supply of $0.9 \mathrm{~V}$, this is not necessary because the signal levels are very low. Leaving out the transformer decreases the chip area and eases the layout. 


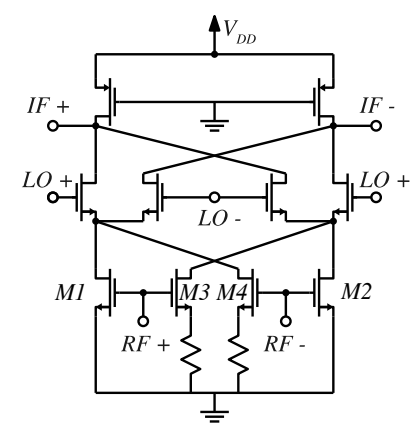

(a)

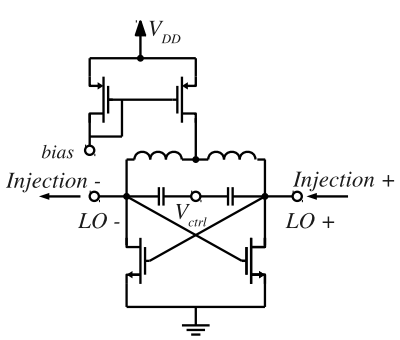

(b)
Fig. 10 Schematic of the downconversion mixer in (a) and the injection locked oscillator in (b).

\section{3 $87 \mathrm{GHz}$ Injection Locked VCO}

The ILO is shown in Fig. 10b. It is an LC oscillator, designed to work at $90 \mathrm{GHz}$, based on [9], and to lock onto the incoming signal to cover dynamic frequency fluctuations in the transmitter VCO. The control voltage can be adjusted to compensate for design errors that result in static frequency deviations.

\subsection{Antenna}

The antenna is the interface between the plastic waveguide and the receiver circuit. A dipole antenna is made by two bondwires, because this results in lower loss than a dipole fabricated in the top-metal [10]. A plane in the top-metal acts as a reflector and shield underneath the antenna. The antenna and shield were simulated in HFSS, together with the plastic waveguide. The antenna is optimized for maximum power transfer from the $E_{11}^{y}$ mode of the waveguide to the chip at $90 \mathrm{GHz}$. The total chip area occupied by the antenna and the shield is $1.1 \mathrm{~mm}^{2}$, which is $52 \%$ of the total chip area. The area underneath the shield can however be used to place digital circuits or analog baseband circuits.

\section{Measurements}

The chip is fabricated in a $40 \mathrm{~nm}$ standard CMOS process. It is glued and wire-bonded to an FR-4 substrate. A chip photograph is shown in Fig. 11.

One end of a $2.2 \mathrm{~mm}$ by $0.9 \mathrm{~mm}$ PP waveguide is inserted into a metal waveguide to apply the RF signal. The other end is brought close to the bondwire antenna on the chip. This is shown in Fig. 12.

The RF signal is generated with an Agilent E8257D PSG combined with an OML S12MS source module. For measurements without modulated signals, the plastic waveguide is connected directly to the output of the source module.

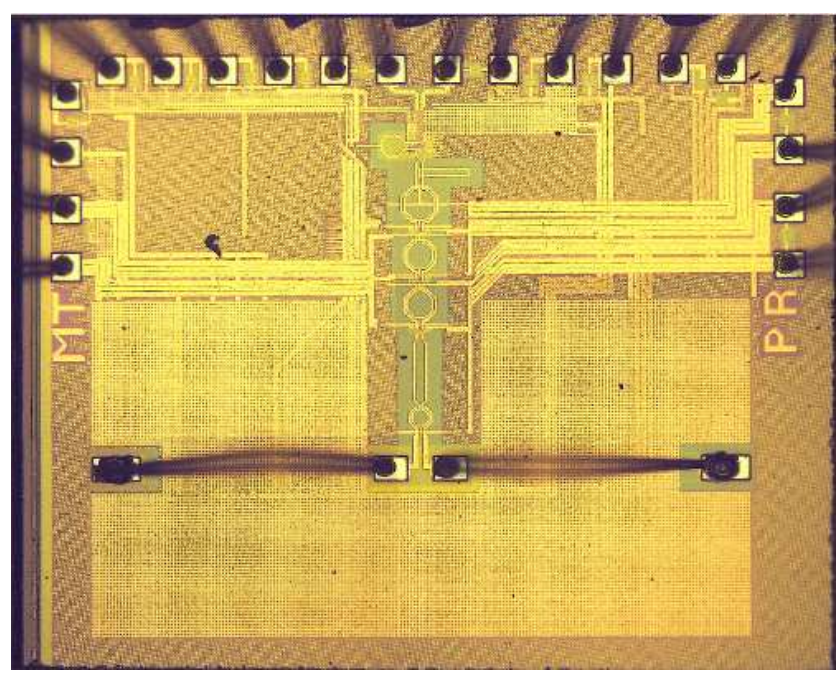

Fig. 11 Micrograph of the chip with the on-chip bondwire antenne at the bottom.

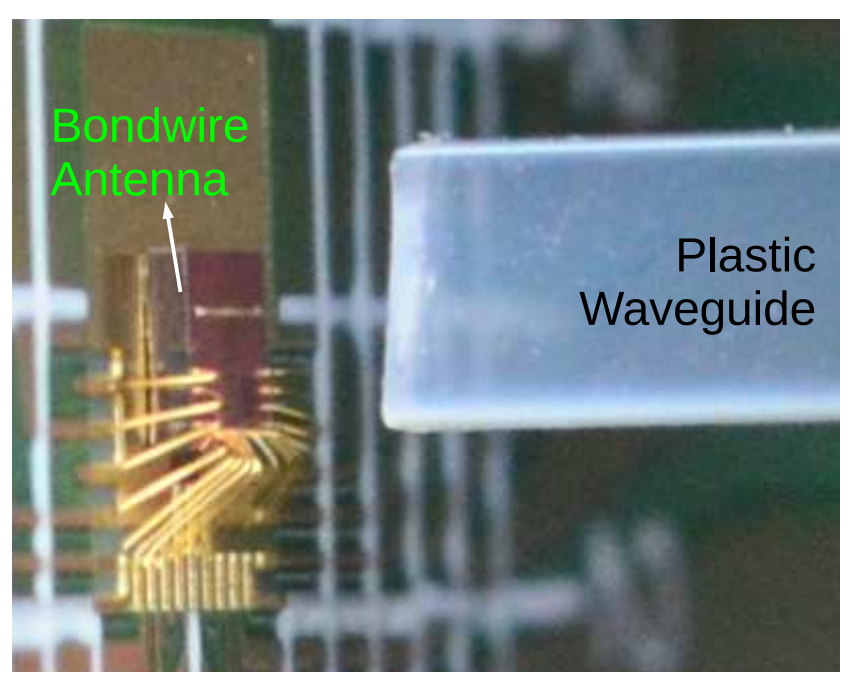

Fig. 12 Chip wire-bonded to PCB with end of plastic waveguide.

When modulated signals are needed, the setup in Fig. 14 is used.

\subsection{Chip Characterization}

The total power consumption is $50 \mathrm{~mW}$ from a $0.9 \mathrm{~V}$ supply, which includes the DC power of the buffers that drive the off-chip $50 \Omega$ impedance of the measurement equipment. The total area, including decoupling capacitors, antenna, reflector and bondpads, is $2.1 \mathrm{~mm}^{2}$. The active area is only $0.21 \mathrm{~mm}^{2}$.

The tuning range of the ILO is measured using a spectrum analyzer with an external mixer and horn antenna to pick up the weak signal from the ILO directly. The tuning range for a control voltage of $-1 \mathrm{~V}$ to $1 \mathrm{~V}$ is $89.5 \mathrm{GHz}$ to $83.1 \mathrm{GHz}$, as can be seen in Fig. 13a. Once the free running frequency of the ILO is set by the control voltage $V_{c t r l}$, the actual fre- 


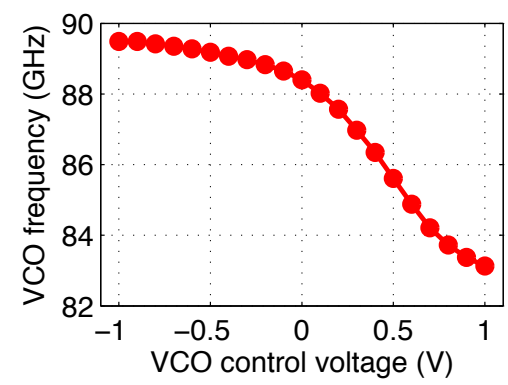

(a) Tuning range of the ILO.

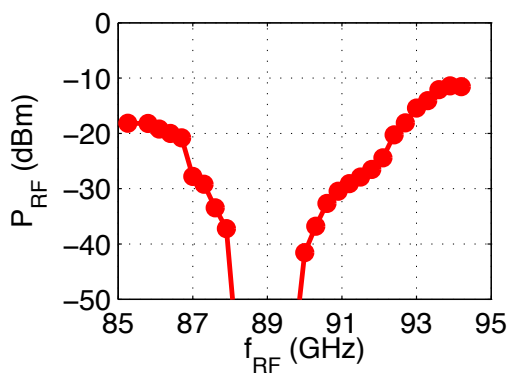

(b) Locking range of the ILO, $V_{c t r l}=0 \mathrm{~V}$.

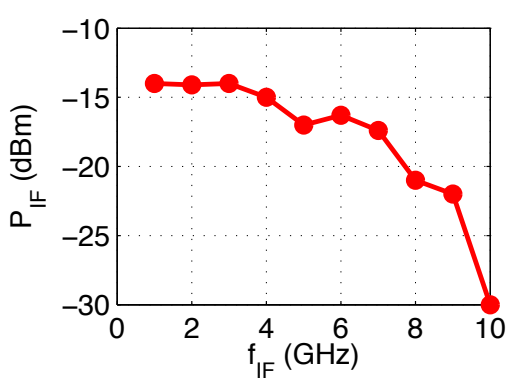

(c) Baseband frequency response.

Fig. 13 Measured performances of the receiver: (a) tuning range, (b) locking range and (c) baseband frequency response.

quency is determined by the locking behaviour. When the input signal to the receiver is large enough, the ILO will lock on the carrier. The more the RF frequency deviates from the free running frequency, the higher this level must be in order for the ILO to lock.

The locking behaviour of the ILO can be examined by applying an RF signal and looking at the IF response on a spectrum analyzer. As long as the ILO is locked to the RF input signal, there's only a DC voltage at the output, but as soon as lock is lost, the difference frequency between the LO and the RF signal is visible on the spectrum analyzer. For each $\mathrm{RF}$ frequency, the input power is lowered untill lock is lost. This power is marked on Fig. 13b. On the $y$-axis, the power coming out of the plastic waveguide is indicated. From the graph, it is seen that lock is most easily achieved at the free running frequency of the ILO.

In a complete link, the control voltages of the oscillators in the transmitter and receivers can be used to bring both fre- quencies close to each other. This is necessary to compensate for process variations or unexpected parasitics, which influence the oscillator frequency. Once in operation, the injection locking will make sure that the receiver oscillator tracks the frequency of the transmitter VCO.

Although the free running frequency of the ILO is $88.4 \mathrm{GHz}$ for $V_{c t r l}=0 \mathrm{~V}$, the highest IF response is measured for an RF frequency of $87 \mathrm{GHz}$. Therefore, the measurements reported next, are performed at that RF frequency.

The baseband frequency response (Fig. 13c) of the chip is measured by applying an $87 \mathrm{GHz}$ RF signal that is amplitude modulated with a sine wave of which the frequency is increased. The on-chip mixer down-converts this signal to baseband. The resulting sine wave is measured with a spectrum analyzer. The baseband bandwidth of $7 \mathrm{GHz}$ is partly limited by the bondwires and the PCB copper traces.

\subsection{Modulated Signals and Complete Link Measurements}

For the generation of Gbps modulated signals at $90 \mathrm{GHz}$, the setup of Fig. 14 is used. The millimeter wave signal from the source module is amplified to drive the LO port of a 80-90 GHz mixer. The baseband signal comes either from a Tektronix AWG7122B arbitrary waveform generator (AWG) or an Agilent 81250 parallel bit error rate tester (ParBERT). An attenuator is used to set the desired amplitude level. The power that comes out of the metal waveguide is measured with an Erickson PM4 power meter. Unless stated otherwise, this level is set to $0 \mathrm{dBm}$, a value that is certainly feasible to be generated with modern CMOS processes [11, $6]$.

Multilevel ASK was demonstrated using the AWG but BER measurements could not be performed [3]. An eye diagram is shown in Fig. 15

For ASK signals, the receiver locks on the LO feedthrough that occurs in the upconversion mixer of the measurement

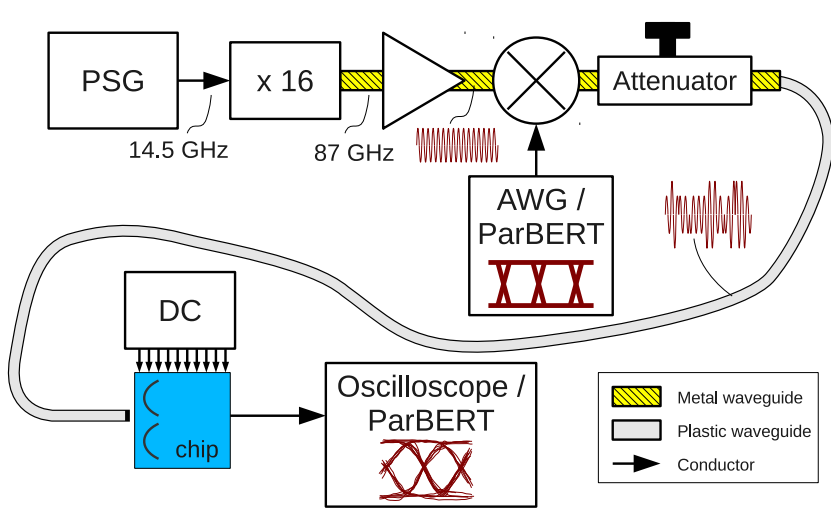

Fig. 14 Measurement setup for data rate testing. A flexible plastic waveguide connects the modulated signal generator to the receiver chip. 


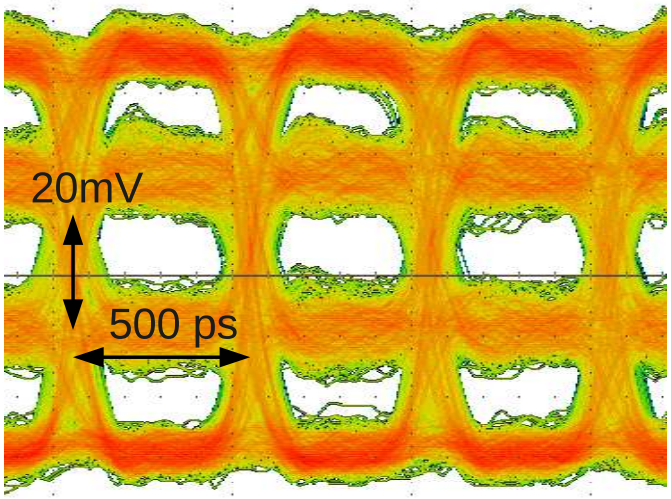

Fig. 15 Eye diagram of a four level ASK signal of $4 \mathrm{Gbit} / \mathrm{s}$, demodulated by the chip.

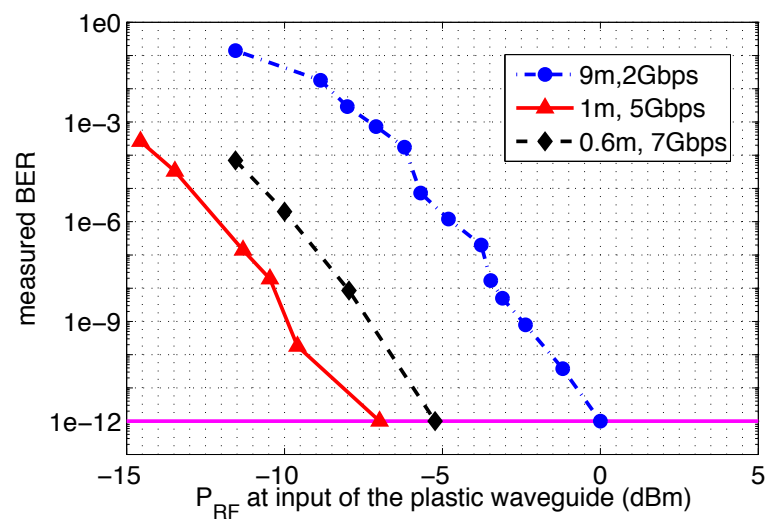

Fig. 16 Measured BER versus input power to the polypropylene waveguide for different data rates and waveguide lengths at $87 \mathrm{GHz}$ carrier frequency.

setup. The ParBERT was used to test bit error rates. For all BER measurements, a PRBS length of $2^{7}-1$ is used. Different lengths of plastic waveguide are tested to determine the influence of the channel, namely power loss and dispersion, on the signal quality. The attenuator is used to control the intput power to the plastic waveguide. The results of three different waveguide lengths and three data rates can be found in Fig. 16. When a confidence level of $95 \%$ is reached for a bit error rate less than $10^{-12}$ [12], the measurement is stopped in order to avoid excessive measurement times.

The maximum data rate measured for a BER $<10^{-12}$ is $9 \mathrm{Gbit} / \mathrm{s}$, over a distance of $60 \mathrm{~cm}$ (not indicated on the graph). The energy efficiency of the receiver is then $5.6 \mathrm{pJ} / \mathrm{bit}$. For a distance of $9 \mathrm{~m}$, the maximum bitrate measured for BER $<$ $10^{-12}$ is $2.5 \mathrm{Gbit} / \mathrm{s}$ (not indicated on the graph). The energy efficiency of the receiver is then $20 \mathrm{pJ} / \mathrm{bit}$. The energy per bit per meter is $2.2 \mathrm{pJ} / \mathrm{bit} / \mathrm{m}$. For comparison, typical values for copper connections are above $10 \mathrm{pJ} / \mathrm{bit} / \mathrm{m}$ while for single mode fibers, this is below $1 \mathrm{pJ} / \mathrm{bit} / \mathrm{m}[13]$.
Table 1 Performance Summary and Comparison to Other Work.

\begin{tabular}{|c|c|c|c|}
\hline & $\begin{array}{c}\text { [6] } 57 \mathrm{GHz} \\
\mathrm{Rx}\end{array}$ & $\begin{array}{l}\text { [14] } 60 \mathrm{GHz} \\
\mathrm{Rx}\end{array}$ & This work \\
\hline $\begin{array}{l}\text { Carrier } \\
\text { quency }\end{array}$ & $57 \mathrm{GHz}$ & $60 \mathrm{GHz}$ & $87 \mathrm{GHz}$ \\
\hline Tx power & $0 \mathrm{dBm}$ & $0 \mathrm{dBm}$ & $0 \mathrm{dBm}$ \\
\hline $\begin{array}{l}\text { Highest bitrate } \\
\text { at distance }\end{array}$ & $\begin{array}{c}15 \mathrm{Gbps} \\
0.1 \mathrm{~m}\end{array}$ & $\begin{array}{c}6 \mathrm{Gbps} \\
2 \mathrm{~m}\end{array}$ & $\begin{array}{c}9 \mathrm{Gbps} \\
0.6 \mathrm{~m}\end{array}$ \\
\hline $\begin{array}{l}\text { Bitrate } \times \text { dis- } \\
\text { tance }\end{array}$ & 1.5 Gbps.m & 12 Gbps.m & 5.4 Gbps.m \\
\hline Longest distance & $1 \mathrm{~m}$ & $7.6 \mathrm{~m}$ & $9 \mathrm{~m}$ \\
\hline Maximum bitrate & $10 \mathrm{Gbps}$ & $3.3 \mathrm{Gbps}$ & $2.5 \mathrm{Gbps}$ \\
\hline $\begin{array}{l}\text { Bitrate } \times \text { dis- } \\
\text { tance }\end{array}$ & $10 \mathrm{Gbps} \cdot \mathrm{m}$ & $\begin{array}{c}25.1 \\
\text { Gbps } \cdot \mathrm{m}\end{array}$ & $22.5 \mathrm{Gbps} \cdot \mathrm{m}$ \\
\hline DC power* & $42 \mathrm{~mW}$ & $16 \mathrm{~mW}$ & $50 \mathrm{~mW}$ \\
\hline Best FOM* & $4.2 \mathrm{pJ} / \mathrm{bit} / \mathrm{m}$ & $0.64 \mathrm{pJ} / \mathrm{bit} / \mathrm{m}$ & $2.2 \mathrm{pJ} / \mathrm{bit} / \mathrm{m}$ \\
\hline Supply & $1.1 \mathrm{~V}$ & $1 \mathrm{~V}$ & $0.9 \mathrm{~V}$ \\
\hline Modulation & ASK & ASK & $\begin{array}{c}\text { (multilevel) } \\
\text { ASK }\end{array}$ \\
\hline Active area* & $0.14 \mathrm{~mm}^{2}$ & $0.08 \mathrm{~mm}^{2}$ & $0.21 \mathrm{~mm}^{2}$ \\
\hline Antenna & $\begin{array}{l}\text { Quasi-Yagi } \\
\text { on PCB }\end{array}$ & $\begin{array}{c}\text { Dipole } \\
\text { off-chip } \\
\text { bondwire }\end{array}$ & $\begin{array}{l}\text { Dipole on-chip } \\
\text { bondwire }\end{array}$ \\
\hline Technology & $\begin{array}{l}40 \mathrm{~nm} \\
\text { CMOS }\end{array}$ & $\begin{array}{l}65 \mathrm{~nm} \\
\text { CMOS }\end{array}$ & $\begin{array}{l}40 \mathrm{~nm} \\
\text { CMOS }\end{array}$ \\
\hline $\begin{array}{l}\text { Plastic Waveg- } \\
\text { uide }\end{array}$ & $\begin{array}{l}\text { Rectangular } \\
8 \times 0.8 \mathrm{~mm}^{2}\end{array}$ & $\begin{array}{l}\text { Hollow } \\
\text { circular } \\
\varnothing 3.2 \mathrm{~mm}\end{array}$ & $\begin{array}{l}\text { Rectangular } \\
2.2 \times 0.9 \mathrm{~mm}^{2}\end{array}$ \\
\hline
\end{tabular}

* Receiver only

\section{Conclusion}

This paper presents a plastic waveguide link with an $87 \mathrm{GHz}$ injection-locked multilevel ASK receiver in 40-nm CMOS with on-chip bondwire antenna. First, the wave propagation in the rectangular dielectric waveguide was analyzed in detail. Simulations of the dielectric loss were confirmed by measurements. Bending loss was also measured for different bending radii. This information was used to select the carrier frequency of the millimeter wave data link.

The performance of the receiver chip was measured and reported. The plastic waveguide link was demonstrated with bitrates up to $9 \mathrm{Gbit} / \mathrm{s}$ for a distance of $60 \mathrm{~cm}$ and $2.5 \mathrm{Gbit} / \mathrm{s}$ for a distance of $9 \mathrm{~m}$. Table 1 summarizes the performance and gives a comparison to other work. The used figure of merit is the energy per bit per meter. In [6], the authors propose a full-duplex interconnect using two links at different frequencies in the same rectangular plastic waveguide. The $57 \mathrm{GHz}$ receiver has a better FOM than the $80 \mathrm{GHz}$ receiver, but it's higher than the one in this work, because their maximum link distance is only $1 \mathrm{~m}$. In [14], a hollow circular waveguide with an outer diameter of $3.2 \mathrm{~mm}$ is used. The loss of this cable is about $1 \mathrm{~dB} / \mathrm{m}$ at $60 \mathrm{GHz}$ which is bet- 
ter than the $1.5 \mathrm{~dB} / \mathrm{m}$ at $90 \mathrm{GHz}$. Thanks to the lower power consumption, the FOM is lower than in this work. This paper however presents the highest carrier frequency in plastic waveguides with the smallest cross-section and longest link length.

The results in this paper support the claim that plastic waveguides can be used for Gbps data links with lengths of $10 \mathrm{~m}$ and more.

Acknowledgements This research is partly supported by the ERC Advanced Grant 227680 (DARWIN).

\section{References}

1. Huber + Suhner, "Datasheet: Coaxial cable: Ez_47_cu_tp," Jan. 2011.

2. Millimeter wave products Inc., "Datasheet: 691 series unflanged waveguides," http://miwv.com/drawings/691/MIWV_ Series691.pdf.

3. M. Tytgat and P. Reynaert, "A plastic waveguide receiver in 40nm CMOS with on-chip bondwire antenna," in Proceedings of the IEEE European Solid-State Circuits Conference (ESSCIRC), 2013, pp. 335-338.

4. E. Marcatili, "Dielectric rectangular waveguide and directional coupler for integrated optics," Bell System Technical Journal, vol. 48, no. 7, pp. $2071-2102$, Sep. 1969.

5. A. Hofmann, E. Horster, J. Weinzierl, L.-P. Schmidt, and H. Brand, "Flexible low-loss dielectric waveguides for $\mathrm{THz}$ frequencies with transitions to metal waveguides," in European Microwave Conference (EuMC), vol. 3, Oct. 2003, pp. 955 - 958.

6. S. Fukuda, Y. Hino, S. Ohashi, T. Takeda, H. Yamagishi, S. Shinke, K. Komori, M. Uno, Y. Akiyama, K. Kawasaki, and A. Hajimiri, "A $12.5+12.5 \mathrm{~Gb} / \mathrm{s}$ full-duplex plastic waveguide interconnect," IEEE Journal of Solid-State Circuits, vol. 46, no. 12, pp. 3113 -3125, Dec. 2011.

7. M. Tytgat, M. Steyaert, and P. Reynaert, "A 186 to $212 \mathrm{GHz}$ downconverter in $90 \mathrm{~nm}$ CMOS," Springer Journal of Infrared, Millimeter, and Terahertz Waves, vol. 33, no. 11, pp. 1085-1103, 2012.

8. N. Deferm and P. Reynaert, "A $100 \mathrm{GHz}$ transformer-coupled fully differential amplifier in $90 \mathrm{~nm}$ CMOS," in IEEE Radio Frequency Integrated Circuits Symposium (RFIC), 2010, pp. 359-362.

9. W. Volkaerts, M. Steyaert, and P. Reynaert, " $118 \mathrm{GHz}$ fundamental VCO with $7.8 \%$ tuning range in $65 \mathrm{~nm} \mathrm{CMOS,"} \mathrm{in} \mathrm{IEEE} \mathrm{Radio}$ Frequency Integrated Circuits Symposium (RFIC), Jun. 2011, pp. 1 -4 .

10. U. Johannsen, A. Smolders, J. Leiss, and U. Gollor, "Bond-wires: Readily available integrated millimeter-wave antennas," in European Microwave Conference (EuMC), Nov. 2012, pp. 197-200.

11. N. Deferm and P. Reynaert, "A $120 \mathrm{GHz} 10 \mathrm{~Gb} / \mathrm{s}$ phase-modulating transmitter in 65nm LP CMOS," in IEEE International Solid-State Circuits Conference, Digest of Technical Papers (ISSCC), Feb. 2011, pp. $290-292$.

12. Maxim, "Statistical confidence levels for estimating error probability," http://notes-application. abcelectronique.com/ 003/3-5321.pdf, 2010.

13. A. Krishnamoorthy, K. Goossen, W. Jan, X. Zheng, R. Ho, G. Li, R. Rozier, F. Liu, D. Patil, J. Lexau, H. Schwetman, D. Feng, M. Asghari, T. Pinguet, and J. Cunningham, "Progress in low-power switched optical interconnects," Selected Topics in Quantum Electronics, IEEE Journal of, vol. 17, no. 2, pp. 357-376, 2011.

14. Y. Kim, L. Nan, J. Cong, and M.-C. Chang, "High-speed mm-wave data-link based on hollow plastic cable and CMOS transceiver," Microwave and Wireless Components Letters, IEEE, vol. 23, no. 12, pp. 674-676, 2013. 
Maarten Tytgat was born in Leuven, Belgium, in 1986. In 2009 he received the degree of M.S. in Electrical Engineering from the KU Leuven, Belgium. He obtained a $\mathrm{PhD}$ degree in electronics at the MICAS laboratories of the KU Leuven on the subject of millimeter wave CMOS receivers. Currently he is working as an Analog and Mixed-Signal ASIC researcher at Alcatel-Lucent Bell Labs.

Niels Van Thienen was born in Turnhout, Belgium, in 1989. In 2012 he received the degree of M.S. in Electrical Engineering from the KU Leuven, Belgium. The subject of his MS thesis was on the design of a fully-integrated 130-nm CMOS class-E resonant inverter for use in DC-AC power converters. He is currently working as a research assistant at the MICAS laboratories of the KU Leuven towards a $\mathrm{PhD}$ degree on millimeter-wave CMOS circuits with a focus on data communication through dielectric waveguides.

Patrick Reynaert was born in Wilrijk, Belgium, in 1976. He received the Master of Industrial Sciences in Electronics (ing.) from the Karel de Grote Hogeschool, Antwerpen, Belgium in 1998 and both the Master of Electrical Engineering (ir.) and the Ph.D. in Engineering Science (dr.) from the University of Leuven (KU Leuven), Belgium in 2001 and 2006 respectively. During 2006-2007, he was a postdoctoral researcher at the Department of Electrical Engineering and Computer Sciences of the University of California at Berkeley. During the summer of 2007, he was a visiting researcher at Infineon, Villach, Austria. Since October 2007, he is an Associate Professor at the University of Leuven (KU Leuven), department of Electrical Engineering (ESAT) and a staff member of the ESAT-MICAS research group. His main research interests include mm-wave and $\mathrm{THz}$ CMOS circuit design, high-speed circuits and RF power amplifiers. Patrick Reynaert is a Senior Member of the IEEE and chair of the IEEE SSCS Benelux Chapter. He serves or has served on the technical program committees of several international conferences including the ISSCC-SRP, IEDM, ESSCIRC and PRIME. He has served as Associate Editor for Transactions on Circuits and Systems I, and as Guest Editor for the Journal of Solid-State Circuits. He received the 2011 TSMC-Europractice Innovation Award and the ESSCIRC-2011 Best Paper award. 